\title{
Exploring Hypersonic, Unstructured-Grid Issues through Structured Grids
}

\author{
Ali R. Mazaheri*and Bil Kleb ${ }^{\dagger}$
}

\begin{abstract}
Pure-tetrahedral unstructured grids have been shown to produce asymmetric heat transfer rates for symmetric problems. Meanwhile, two-dimensional structured grids produce symmetric solutions and as documented here, introducing a spanwise degree of freedom to these structured grids also yields symmetric solutions. The effects of grid skewness and other perturbations of structured-grids are investigated to uncover possible mechanisms behind the unstructured-grid solution asymmetries. By using controlled experiments around a known, good solution, the effects of particular grid pathologies are uncovered.

These structured-grid experiments reveal that similar solution degradation occurs as for unstructured grids, especially for heat transfer rates. Non-smooth grids within the boundary layer is also shown to produce large local errors in heat flux but do not affect surface pressures.
\end{abstract}

\section{Introduction}

$\mathrm{H}^{2}$ YPERSONIC unstructured-grid development has attracted considerable attention due to the complexity of generating and adapting structured grids. Despite the advantages of unstructured grids, several issues with unstructured-grid techniques have been reported. ${ }^{1-3}$ Gnoffo and White ${ }^{4}$ showed that aerothermodynamic computations over an axisymmetric geometry with a pure-tetrahedral unstructured grid, result in asymmetric predictions in the spanwise direction and across the symmetry plane. In their study, a uniformly biased unstructured grid was generated from a structured grid and the surface heat transfer rates were compared with a structured grid solution. The unstructured grid and solution from Reference 4 are shown in figure 1.

Nompelis et al. ${ }^{5}$ developed a hybrid implicit method for the solution of compressible Navier-Stokes equations on hybrid unstructured meshes. With this method, flow over a circular cylinder traveling at Mach 17.6 was simulated on several hybrid unstructured meshes. Heat transfer rate predictions were asymmetric for all the hybrid unstructured meshes except for the grid with hexahedral cells within the bow shock region. The surface pressure distributions were, however, symmetric with respect to the stagnation line although spanwise variations were present for all grid cases.

Understanding the fundamental mechanism that causes an unstructured-grid technique to yield such unphysical behavior is essential to developing robust unstructured-grid techniques. While other efforts are focused on unstructured meshes, this study uses structured-grid aerothermodynamic simulations to search for grid characteristics that lead to similar asymmetries. If the effects of the grid are large and unpredictable, a better solver needs to be developed and experience in grid pathologies becomes crucial.

In this paper, structured meshes are first shown to provide a spanwise-symmetric solution for the Mach 17 cylinder case of Gnoffo and White ${ }^{4}$ and then used to mimic characteristics of unstructured meshes. Grid skewness, asymmetries, and high and low frequency perturbations of unstructured grids are all modeled within the structured grid framework. These results are studied to discern which types of perturbations lead to asymmetric predictions.

*AMA, Inc./NASA LaRC, Aerothermodynamics Branch, AIAA Member; Ali.R.Mazaheri@nasa.gov

†NASA Langley Research Center, Aerothermodynamics Branch, Lifetime AIAA Member; Bil.Kleb@nasa.gov 


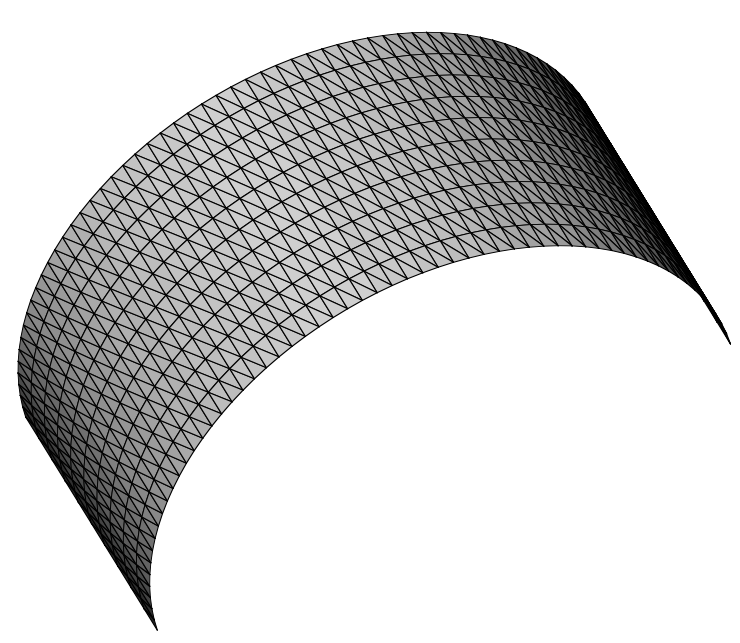

(a) Surface grid.

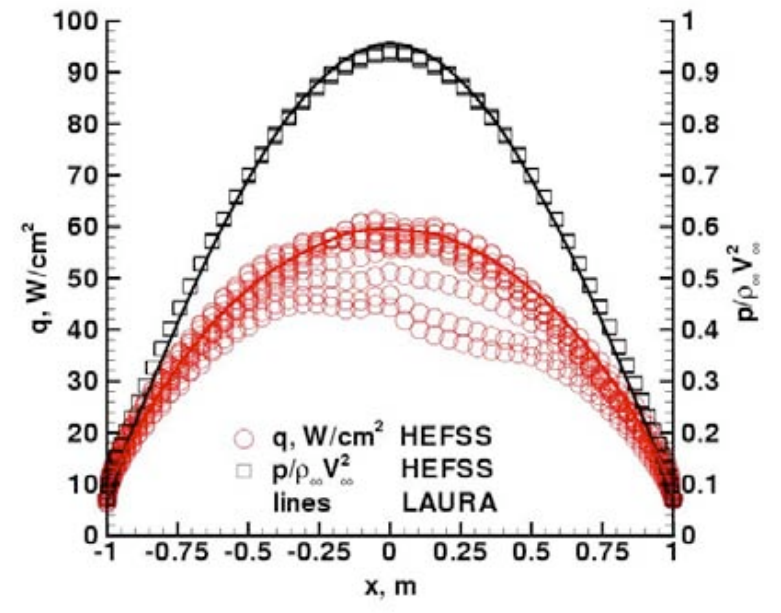

(b) Solution.

Figure 1. Surface pressure and heat transfer rate distributions for a biased unstructured grid (from Ref. 4.)

\section{Computational Modeling}

For this study, the extruded cylinder case of Gnoffo and White ${ }^{4}$ is used for all computational simulations. Perfect gas, laminar air flow is modeled with the structured-grid Langley Aerothermodynamic Upwind Relaxation Algorithm (LAURA) ${ }^{6,7}$ for the forebody of a $1 \mathrm{~m}$ radius cylinder traveling at Mach 17.6 with a Reynolds number of $3.77 \times 10^{6}$. The full Reynolds-Averaged Navier-Stokes equations are iteratively converged until the $L_{2}$ norm of the residuals is $10^{-9}$.

A fully symmetric structured-grid over the $1 \mathrm{~m}$ long extruded cylinder baseline grid is generated by creating a symmetric, bow-shock aligned structured grid on a two-dimensional cylinder and then extruding the grid in a spanwise direction with a constant cell size increment. This baseline grid is shown in Figure 2

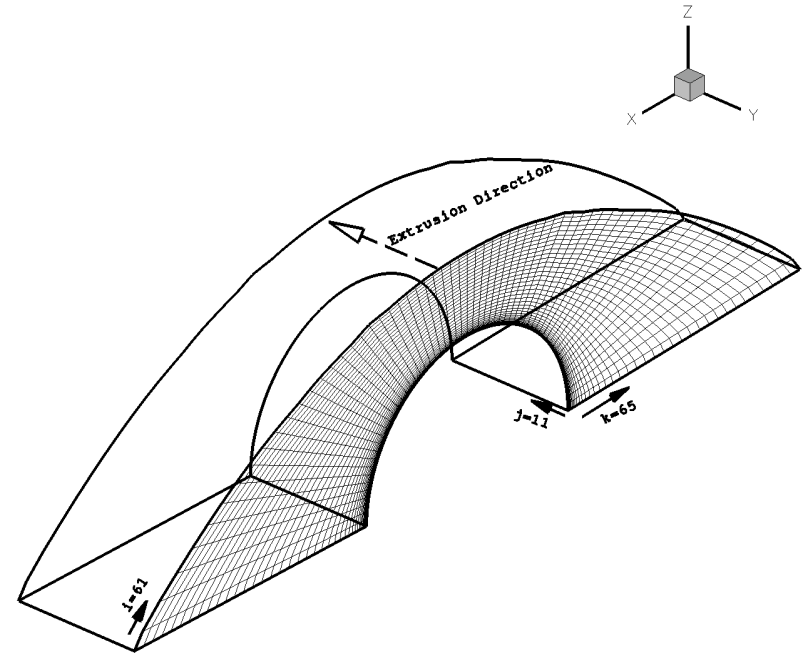

(a) Extrusion/topology.

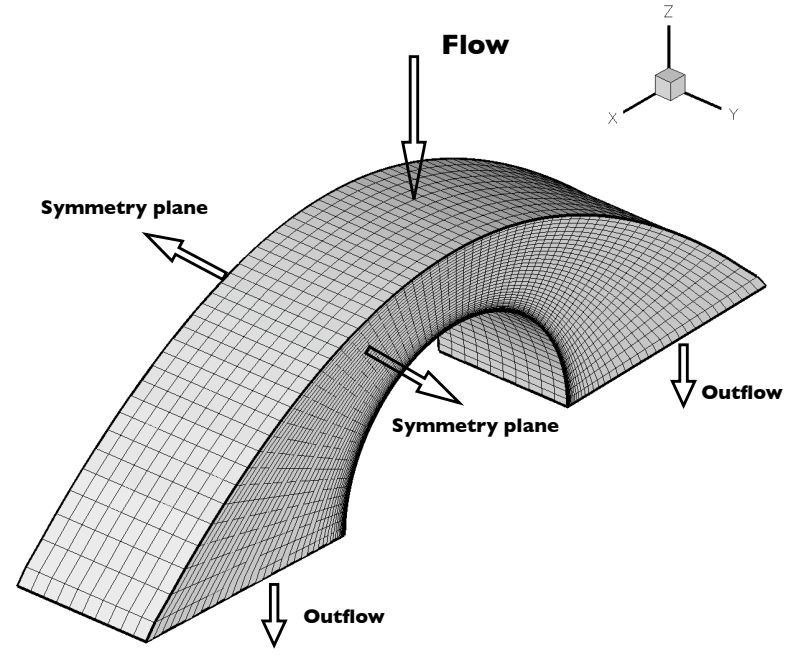

(b) Boundary conditions.

Figure 2. Baseline computational domain.

and contains a total of 38,400 computational cells, $60 \times 10 \times 64$. A no-slip boundary condition is imposed on the solid surface, the outer boundary is specified as free-stream, and symmetry is assigned for the two remaining surfaces. 


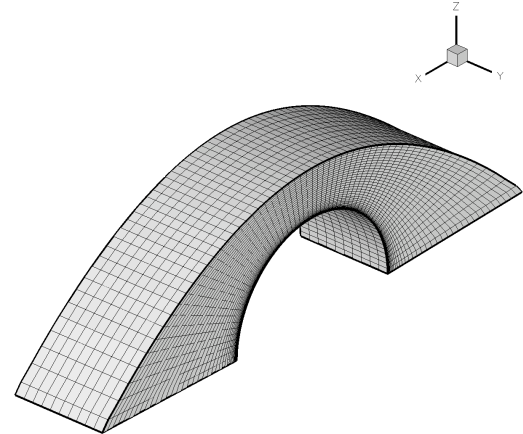

(a) Baseline grid

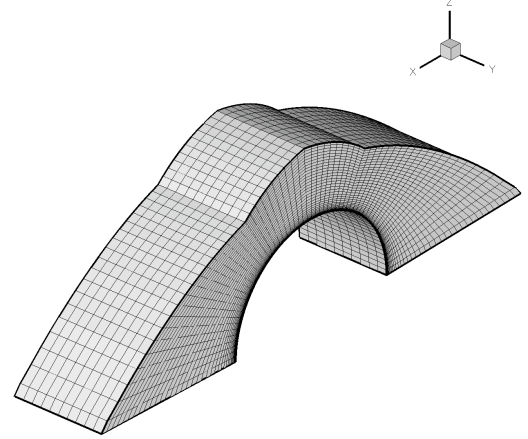

(d) Partial grid misalignment (including $\mathrm{BL})$

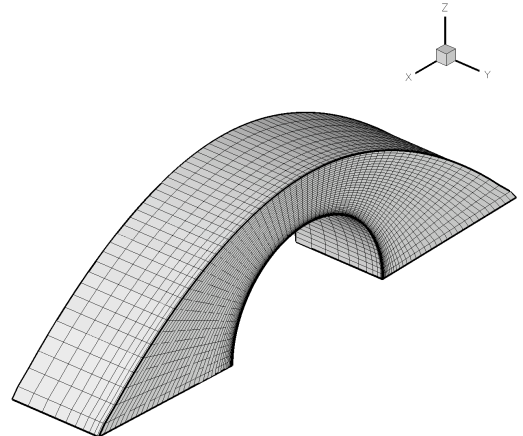

(b) Spanwise stretched grid

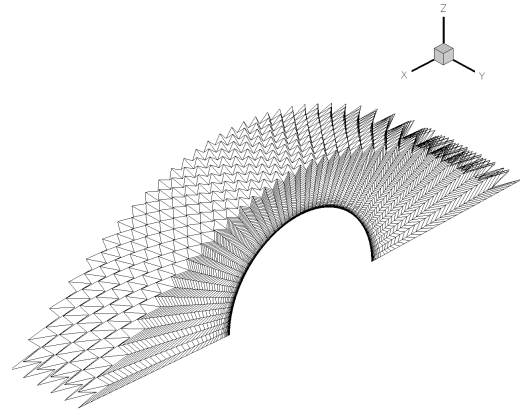

(e) Symmetric irregular \& skewed grid (unmodified BL)

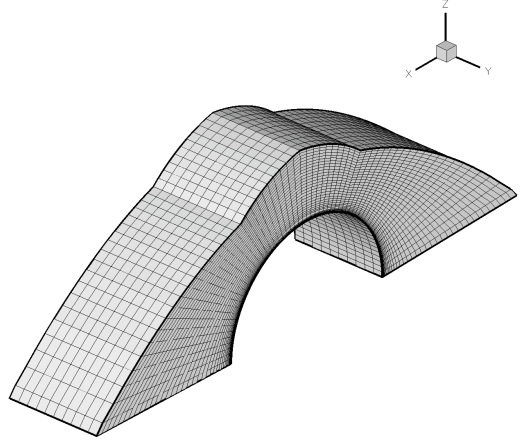

(c) Partial grid misalignment (unmodified BL)

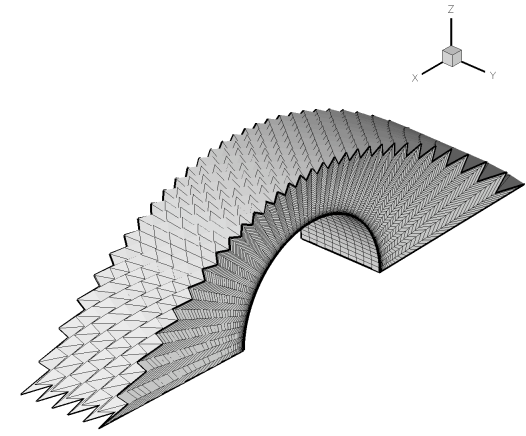

(f) Symmetric irregular \& skewed grid (including BL)

Figure 3. Structured grids perturbations (continued).

This baseline grid is manipulated in several different ways to mimic unstructured-grid characteristics. Figure 3 includes this baseline grid and all the perturbations explored during this study. To reduce repetition in the following discussion, each subfigure corresponds to a case name, e.g., subfigure $3 \mathrm{~b}$ will correspond to Case B, 3c will be known as Case C, and so on. Case A will also be referred to as the baseline case.

Case B has spanwise spacing gradually increased to make the grid anisotropic along this direction. For all remaining cases, the surface grid points are kept the same as the baseline grid.

To determine the affects of grid misalignment with the bow shock, Cases C and D have one third of the computational cells misaligned with bow shock. The misaligned region is centered on the stagnation point, which has been stretched by $50 \%$ and linearly blended back to the baseline grid by the $20^{\text {th }}$ and the $40^{\text {th }}$ cells in the $i$-direction. Spline interpolation is used in the $k$-direction. To separate the effects of computational cell structure within the boundary layer, the first forty cells normal to the surface are frozen in Case C, while the cells within the boundary layer was also modified for Case D using a linear interpolation between the surface grid points and the $40^{\text {th }}$ grid points normal to the surface.

To mimic tetrahedral-grid faces not aligned with the bow shock, high-frequency perturbations are added to the grid coordinates along $k$ lines by alternating arclength stretching factors in the $i$ - and $j$-directions of the baseline case. Cases $\mathrm{E}-\mathrm{G}$ are grids with high-frequency perturbations, while cases $\mathrm{H}-\mathrm{J}$ have perturbation of lower frequency. Cases $\mathrm{E}$ and $\mathrm{F}$ are symmetric in the spanwise direction and across the stagnation line, with unmodified and modified cells within boundary layer regions, respectively. Cases $\mathrm{E}$ and $\mathrm{F}$ are generated by stretching the $k$-arclength by $20 \%$ for every i and $\mathrm{j}$ indicdes. For Case $\mathrm{E}$, the first forty cells that lie within the boundary layer are not altered, while all the grid cells are modified in Case F. Case G is created similar to Case $\mathrm{E}$ with the exception that the acrlength change varies from the $2.5 \%$ to $40 \%$. Case $\mathrm{G}$ is non uniform in the spanwise direction but symmetric with respect to the stagnation line. 


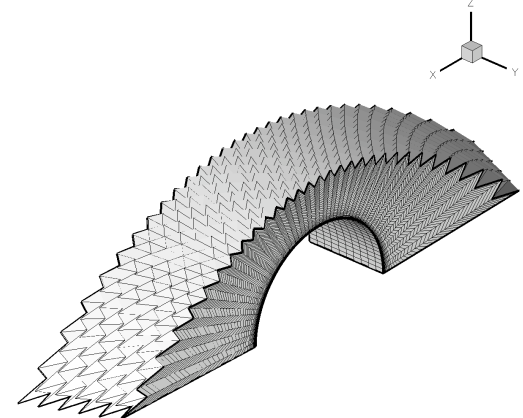

(g) Asymmetric irregular \& skewed grid (unmodified BL)

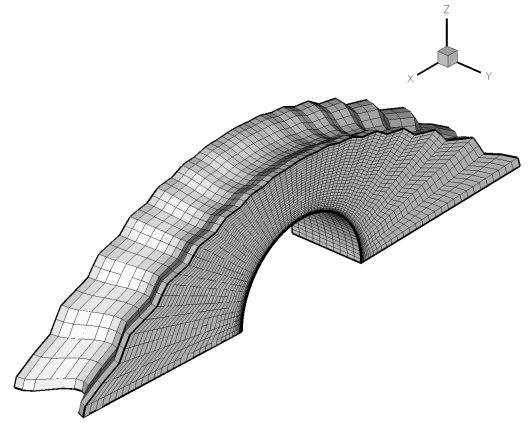

(j) Smoothed asymmetric grid (unmodified BL)

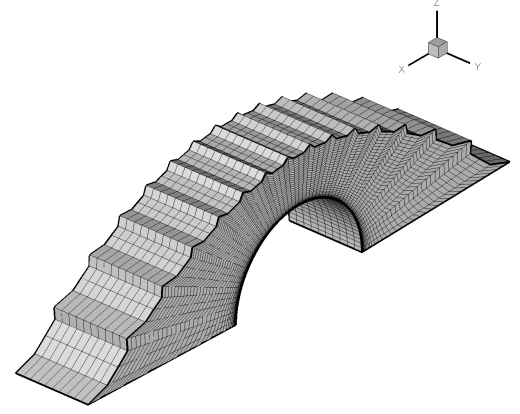

(h) Symmetric saw tooth grid (unmodified $\mathrm{BL}$ )

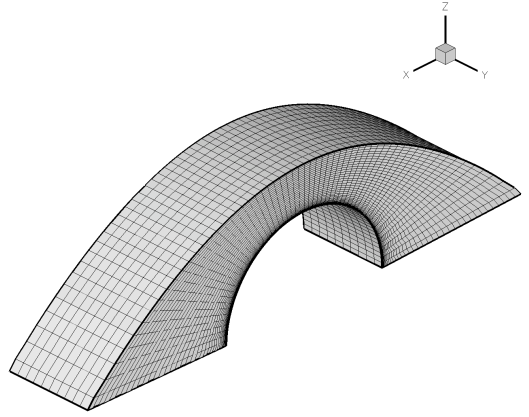

(k) Irregular \& skewed grid (modified BL; fixed outer boundary)

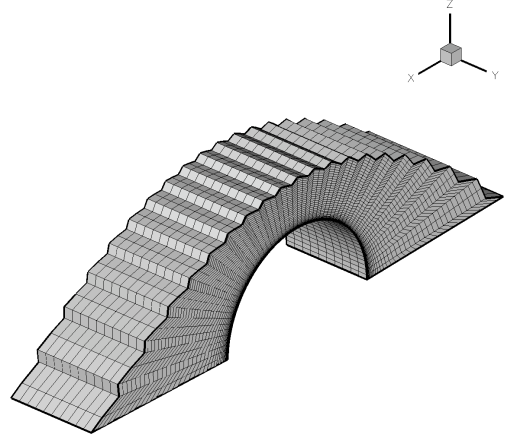

(i) Biased asymmetric saw-tooth grid (unmodified BL)

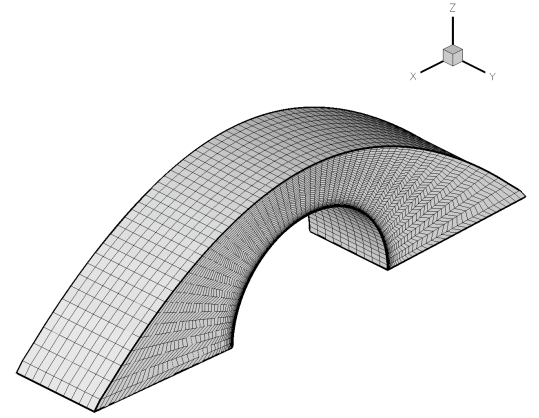

(1) Skewed BL grid with the baseline grid

Figure 3. Structured grids perturbations (concluded).

Case $\mathrm{H}$ is created by increasing the $k$-acrlength of every fourth $i$ index, and of all the $j$ indices by $10 \%$. Case I is similar to the Case $\mathrm{H}$ except that every third $i$ index is altered so that the grid becomes biased asymmetric in $i$-direction. To generate Case $\mathrm{J}$, the $k$-arclength of every other four $i$ indices located on the symmetry planes was increased by $50 \%$. An increase of $25 \%$ was added to the $k$-acrlength at $\mathrm{j}=5$. The grids are then blended using an elliptic interpolation in $i$ - and $j$-directions, and a linear interpolation in $k$-direction.

Case $\mathrm{K}$ is generated by skewing the baseline grid from the surface out to the outer boundary. In this case the outer boundary is kept the same as the baseline grid, and the $k$-arclength of every second $i$ index is increased by $15 \%$. In Case L, however, only the first 30 cells within the BL are modified. This means that the outer boundary and the shock region remain aligned with the bow shock for both cases.

\section{Results and Discussions}

Aerothermodynamic properties including the surface pressure, $p$, surface heat transfer rates, $q$, bow shock thickness, and bow shock stand-off distance are compared with the baseline case shown in Figure 4. As above, the solution on this grid is referred to as the baseline solution and Figure 4b confirms a symmetric solution across the stagnation line. The results of this baseline case are compared against all other grids shown in Figure 3 to search for mechanisms that lead to the asymmetric behavior observed with unstructured-grid meshes.

For all cases, surface pressures and heat transfer rates are compared with the baseline case, in Figure 5. Case B shows that the heat transfer rate distributions remained symmetric with spanwise anisotropy. However, this spanwise-stretched grid under-estimates the surface heat transfer rates by about $4 \%$ at the stagnation point, while surface pressure remains undisturbed.

Case $\mathrm{C}$ shows minor circumferential asymmetries for the heat transfer rate close to the stagnation region, but otherwise spanwise symmetry. 


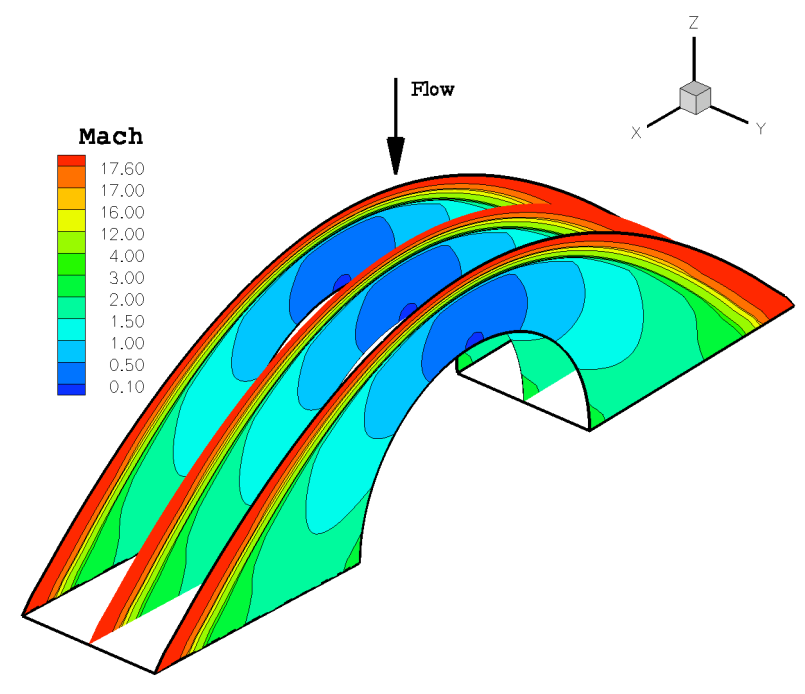

(a) Mach contours.

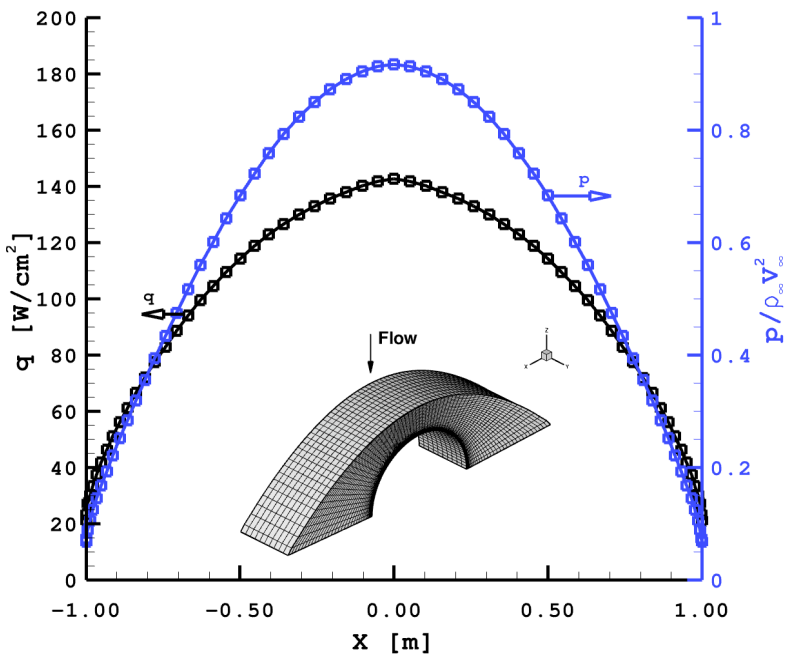

(b) Surface distributions.

Figure 4. Hypersonic flow over extruded cylinder for baseline case of Mach 17.6 and Reynolds number of $3.77 \times 10^{6}$.

Upon also modifying the cells within the boundary layer, however, Case D shows acute asymmetric predictions of the surface heat transfer rates in the circumferential direction, while remaining symmetric in the spanwise direction. Note that the asymmetric behavior only appears in the region where the mesh is misaligned with the bow shock. Case E under-estimates the surface heat transfer rates by $5 \%$ when compared to the baseline solution. For Case F, however, where the grid cells within the boundary layer are also altered, this under-prediction is more severe $(8 \%)$. Note also that both grids yield symmetric circumferential and spanwise solutions. Case $\mathrm{G}$ shows asymmetric surface pressure and heat transfer distributions in spanwise direction. Case G, however, did not produce asymmetric results in the circumferential direction. Comparing the results of Case $\mathrm{G}$ with Cases B-F shows that spanwise grid asymmetries cause asymmetries solutions along the same direction.

Examining Cases $\mathrm{H}-\mathrm{J}$ shows that irregular and skewed structured grids with low frequency irregularities result in predictions that deviate from the baseline solution by $4-12 \%$. Case $\mathrm{H}$ shows that an intermittent symmetric saw-tooth grid causes the heat transfer rates and surface pressure to be higher than that of the baseline case at the stagnation point by $4 \%$, while Case I shows that a combination of low frequency irregular cells and asymmetric properties in circumferential direction lead to asymmetric computational predictions.

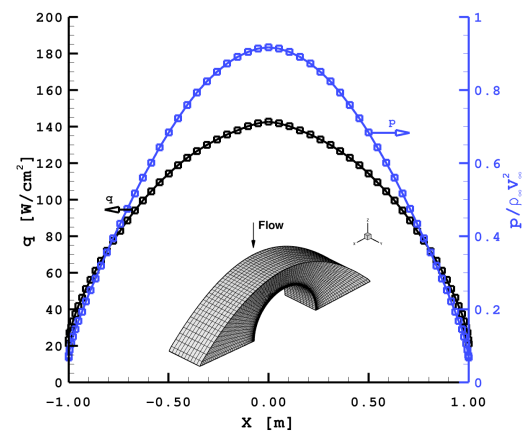

(a) Baseline grid

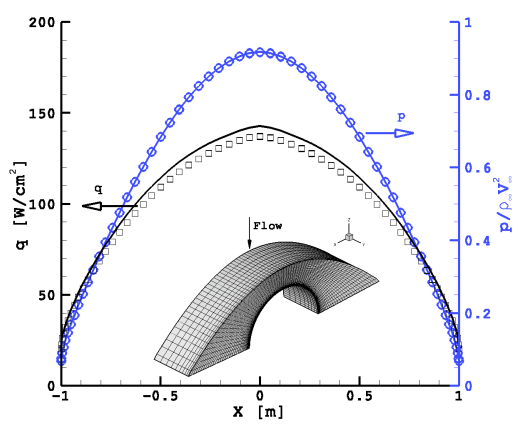

(b) Spanwise stretched grid

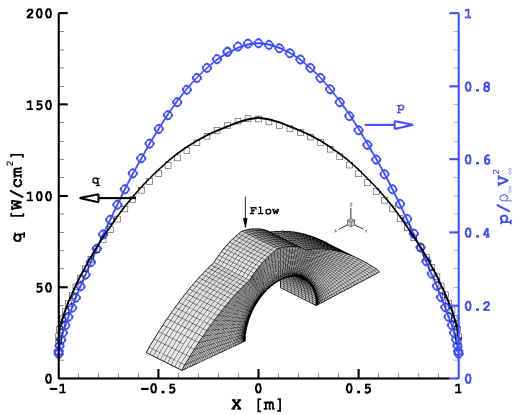

(c) Partial grid misalignment (unmodified BL)

Figure 5. Variations of surface pressure and heat transfer rates for hypersonic flow over extruded cylinder at Mach 17.6 and Reynolds number of $3.77 \times 10^{6}$. The symbols represent the modified grid solutions with each spanwise station over plotted, and the lines are the baseline solution (continued). 
And as before, the asymmetry of the surface heat transfer rates is more pronounced than for the surface pressure.

Case J, having a combination of low frequencies in spanwise direction under-estimates the surface heat transfer rate distributions by as much as $12 \%$. In addition, both pressure and surface heat transfer rates are spanwise asymmetric. Comparing the results of Cases $\mathrm{G}$ and $\mathrm{J}$, which both have asymmetric grids in the spanwise direction, shows that asymmetric grids in spanwise direction lead to spanwise asymmetries.

Cases $\mathrm{K}$ and $\mathrm{L}$ reveal whether the errors associated with the skewed and irregular grids are inherited from the shock or from the boundary layer. Recall that the grid for Case K alters cells within the BL while Case $\mathrm{L}$ leaves them unmodified. Both cases show a 5\%-6\% error in heat transfer rate calculations. So while, grid skewness within the boundary layer creates a large local error in prediction of heat transfer rates (see Case D) the combination of grid irregularities in the shock and BL regions reduces the local error.

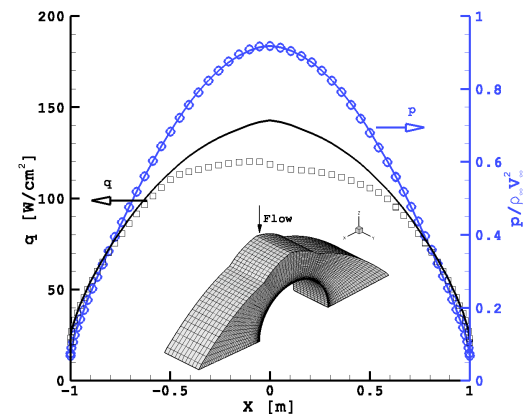

(d) Partial grid misalignment (including $\mathrm{BL})$

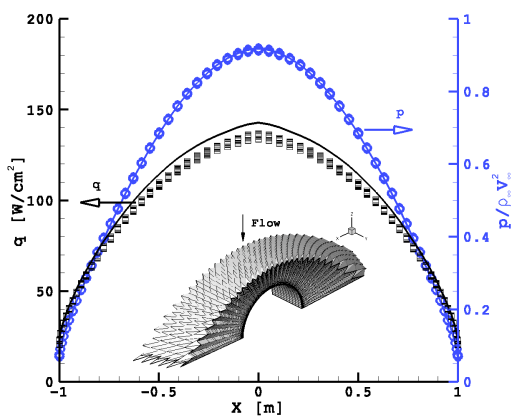

(g) Asymmetric irregular \& skewed grid (unmodified BL)

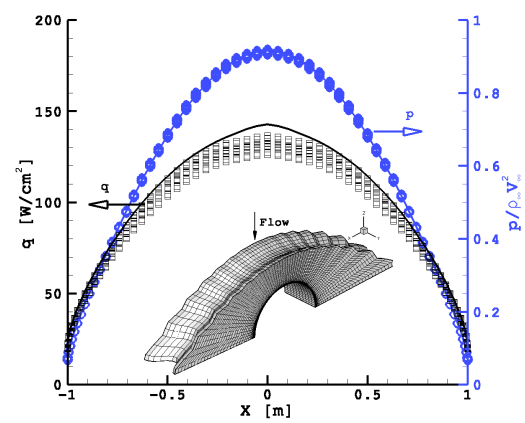

(j) Smoothed asymmetric grid (unmodified BL)

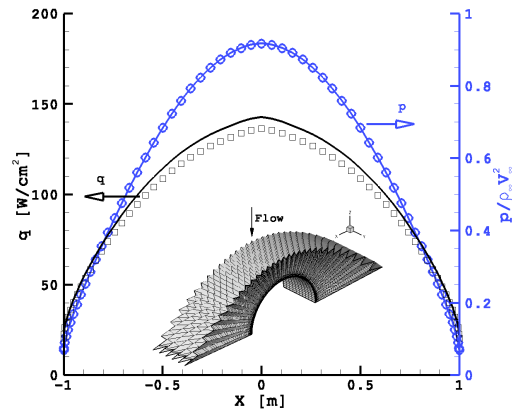

(e) Symmetric irregular \& skewed grid (unmodifiedBL)

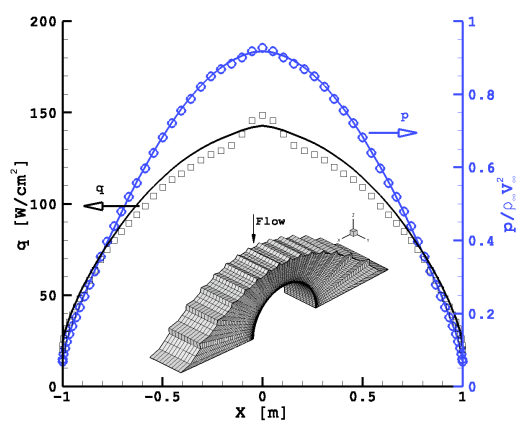

(h) Symmetric saw tooth grid (unmodified BL)

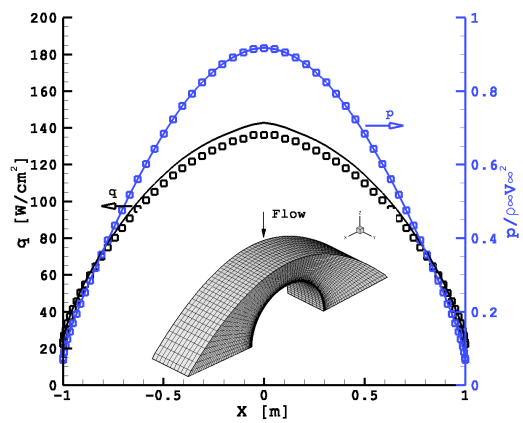

(k) Irregular \& skewed grid (modified BL; fixed outer boundary)

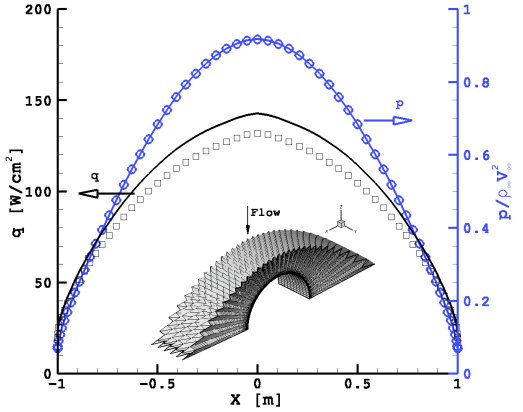

(f) Symmetric irregular \& skewed grid (including BL)

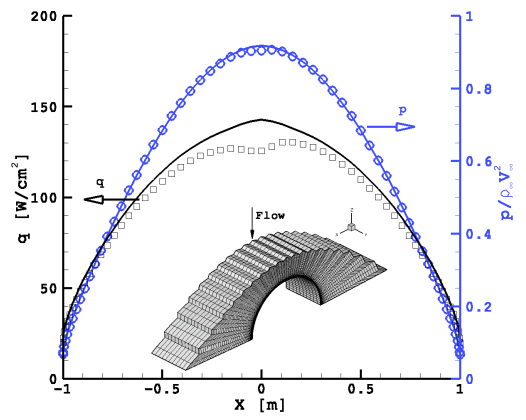

(i) Biased asymmetric saw-tooth grid (unmodified BL)

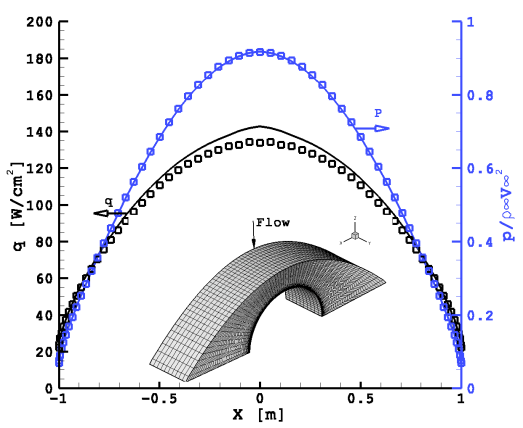

(1) Skewed BL grid with baseline grid

Figure 5. Concluded. 
The bow shock thickness, $\delta / r$, and its stand-off distance, $h / r$, for all the cases are tabulated in Table 1, where the shock thickness is defined as the closest distance between the sonic line and the free-stream mach contour. The tabulated data shows that the shock stand-off distance, $\delta / r$, is about 0.45 , regardless of the grid topology ${ }^{\mathrm{a}}$. The bow shock thickness, however, varies by as much as $70 \%$ when compared with the baseline case. Computational results showed that the bow shock shape is highly affected by the outer boundary grid cells, emphasizing the importance of the grid alignment with the bow shock in accurately predicting the shock and aerothermodynamic properties. However, for all the cases studied except in Cases C and D, the bow shock is confined within two cells. This is due to the one-dimensional operator splitting of the cell-centered shock capturing algorithm. As an example, the bow shock for Case $\mathrm{F}$ is shown in Figure 6.

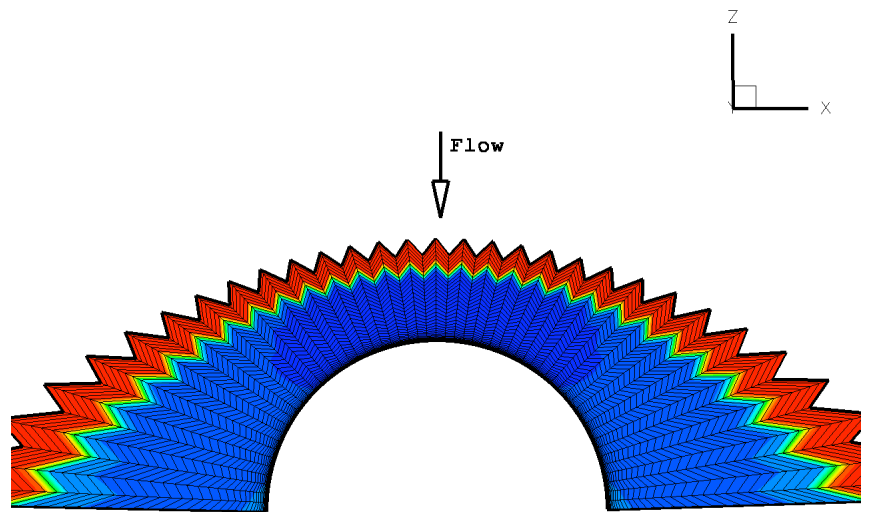

Figure 6. Bow shock structure for Case F.

The claim is often made that grid effects are more serious for heat transfer rate than skin friction. To examine this hypothesis, heat transfer rates and skin friction coefficients, $C_{f}$, are shown in Figure 7 . The grid irregularities affect the heat transfer rate by as much as $18 \%$ but the skin friction deviates from the baseline values by only $6 \%$.

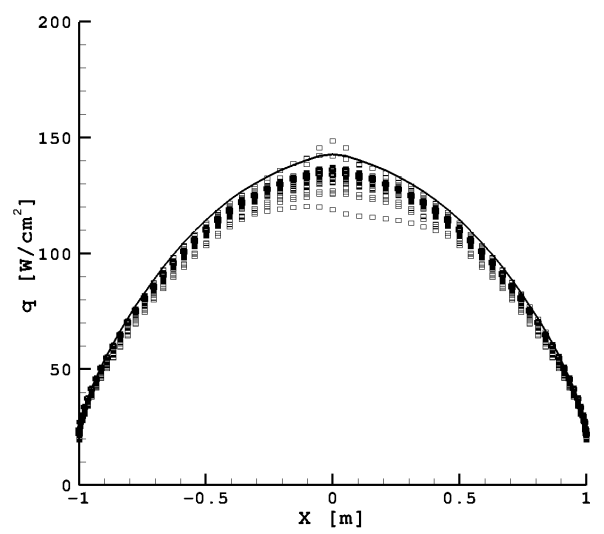

(a) Heat transfer.

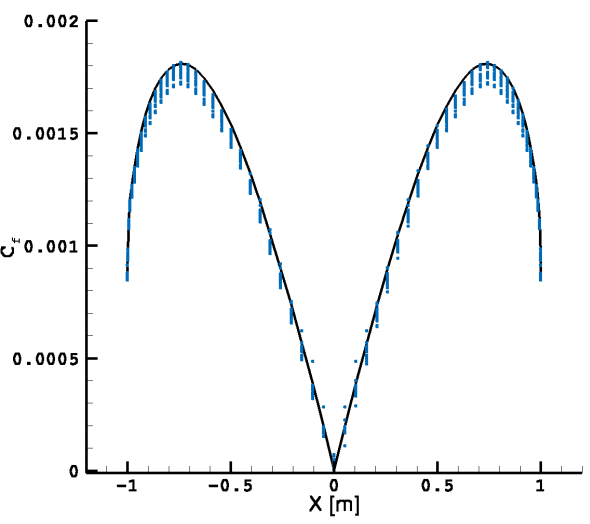

(b) Skin friction.
Table 1. Bow shock thicknesses and stand-off distances.

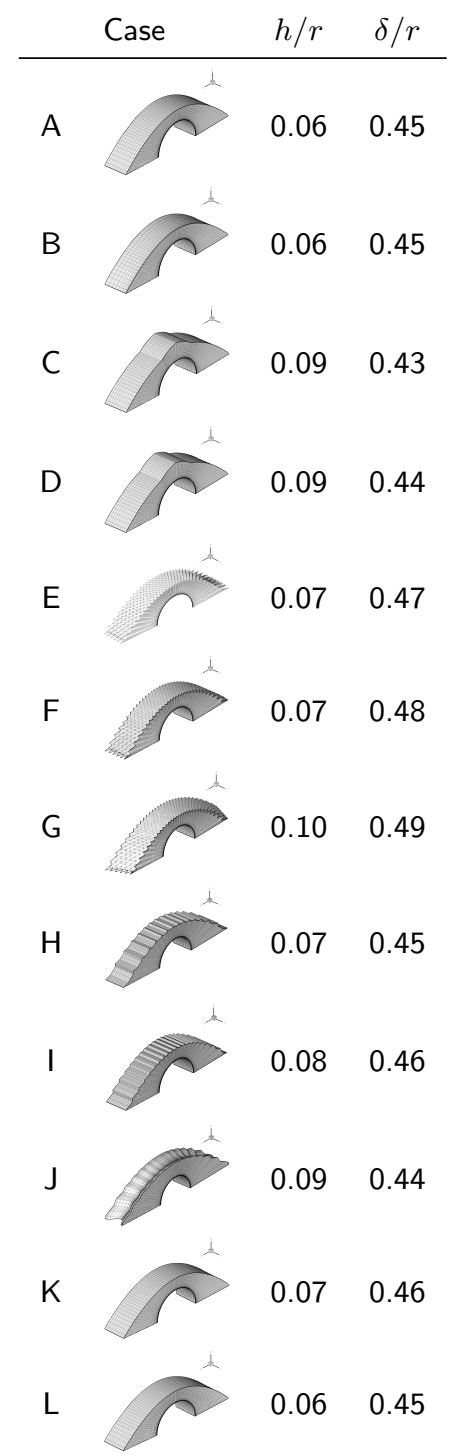

Figure 7. Distribution variations for all cases. The solid lines depict the baseline case while the symbols represent all the results in Figure 5.

\footnotetext{
${ }^{\mathrm{a}}$ The inviscid approximations of Probstein ${ }^{8}$ and Seiff $^{9}$ give 0.42.
} 


\section{Conclusions}

In this paper, mesh sensitivity for structured-grid hypersonic aerothermodynamic simulations were investigated. The analyses were studied with LAURA to investigate the asymmetric issues known for pure tetrahedral unstructured-grid computational aerothermodynamic simulations. Structured-grid meshes were manipulated to mimic unstructured-grid characteristics for blunt body traveling at the Mach 17.6 with a Reynolds number of $3.77 \times 10^{6}$. The results reveal the following pathologies:

- Grid alignment with the bow shock plays an important role in predicting an accurate computational aerothermodynamic results for blunt body hypersonic problems.

- Spanwise anisotropic grid cells under-predict the surface heat transfer rate distributions but do not lead to asymmetric results.

- Highly irregular and skewed grid cells lead to under-prediction of surface quantities, but remain symmetric if the grid is symmetric in the spanwise direction.

- Asymmetric grid cells with low frequencies perturbation result in asymmetric computational results.

- Irregular symmetric grid cells with low frequency irregularities have a greater effect than those with higher frequencies.

- Grid irregularities within the boundary layer lead to large local error in heat transfer rate prediction.

Based on this investigation, the current dimensionally-split flux scheme fails to adequately compensate for grid irregularities and yields to large errors in surface pressure and heat transfer rates. This fuels the ongoing quest for truly multidimensional algorithms.

\section{Acknowledgments}

The authors would like to thank Dr. Peter Gnoffo, Dr. Bill Wood, Bill Jones, and Karen Bibb of NASA Langley, Hampton, VA, and Prof. Phil Roe of University of Michigan, Ann Arbor, for constructive criticism of this work.

\section{References}

\footnotetext{
${ }^{1}$ Barth, T. J., "Numerical Aspects of Computing Viscous High Reynolds Number Flows on Unstructured Meshes," AIAA Paper 91-0721, 1991.

${ }^{2}$ Aftosmis, M., Gaitonde, D., and Tavares, T. S., "Behavior of Linear Reconstruction Techniques on Unstructured Meshes," AIAA Journal, Vol. 33, No. 11, 1995, pp. 2038-2049.

${ }^{3}$ Weinberg, Z. and Long, L. N., "A Massively Parallel Solution of the Three-Dimensional Navier-Stokes Equations on Unstructured, Adaptive Grids," AIAA Paper 94-0760, 1994.

${ }^{4}$ Gnoffo, P. A. and White, J. A., "Computational Aerothermodynamic Simulation Issues on Unstructured Grids," AIAA Paper 2004, 2004-2371.

${ }^{5}$ Nompelis, I., Drayna, T. W., and Candler, G. V., "Development of a Hybrid Unstructured Implicit Solver for the Simulation of Reacting Flows over Complex Geometries," AIAA Paper 2004-2227, 2004.

${ }^{6}$ Gnoffo, P. A., Gupta, R. N., and Shinn, J. L., "Conservation Equations and Physical Models for Hypersonic Air Flows in Thermal and Chemical Nonequilibrium," NASA TP 2867, Feb. 1989.

${ }^{7}$ Gnoffo, P. A., "An Upwind-Biased, Point-Implicit Relaxation Algorithm for Viscous, Compressible Perfect-Gas Flows," NASA TP 2953, Feb. 1990.

${ }^{8}$ Probstein, R. F., "Inviscid Flow in the Stagnation Point Region of Very Blunt-Nosed Bodies at Hypersonic Flight Speeds," WADC TN 56-395, Brown University, 1956.

${ }^{9}$ Seiff, A., "Gasdynamics in Space Exploration," NASA SP 24, 1962.
} 